\title{
224 M2 MACROPHAGE-MEDIATED IMMUNE SUPPRESSION OF CHIMERIC ANTIGEN RECEPTOR T CELLS VIA PD-L1 SIGNALING IN PROSTATE CANCER
}

Yukiko Yamaguchi*, Jackson Gibson, Kevin Ou, Saul Priceman. City of Hope National Medical Center, Duarte, CA, USA

Background The immune suppressive tumor microenvironment (TME) that inhibits $\mathrm{T}$ cell infiltration, survival, and anti-tumor activity has posed a major challenge for developing effective immunotherapies for solid tumors. Chimeric antigen receptor $\mathrm{T}$ cell therapy has shown unprecedented clinical response in treating patients with hematological malignancies, and intense investigation is underway to achieve similar responses with solid tumors. Immunologically cold tumors, including prostate cancers, are often infiltrated with abundant macrophages, and infiltration of M2 macrophages correlates with metastasis and poor prognosis.

Methods To model this in vitro, we utilized a novel co-culture system with tumor cells, prostate stem cell antigen (PSCA)directed CAR T cells, and polarized macrophages. To investigate the TME in vivo, we took advantage of "humanized" MISTRG mice, which are immunocompromised mice with knocked-in human genes that support human hematopoiesis and efficient tumor-infiltration of myeloid cell populations. Humanized MISTRG mice were intratibially engrafted with LAPC9 tumor cells to model bone metastatic disease.

Results We observed significant hampering of PSCA-CAR $\mathrm{T}$ cell activity in vitro with the presence of M2 macrophages, but not M1 macrophages, coinciding with a robust induction of PD-L1 in both tumor cells and macrophages. We also observed PD-L1 expression in tumor-associated macrophages infiltrating tumors following PSCA-CAR T cell therapy in the humanized mice. Anti-PD-L1 monoclonal antibodies in combination with CAR-T cell therapy altered phenotype and survival of M2 macrophages, resulting in improved anti-tumor activity of PSCA-CAR T cells in the presence of M2 macrophages.

Conclusions Recently, immune checkpoint (IC) blockade (ICB) has been utilized in combination with chimeric antigen receptor (CAR) $\mathrm{T}$ cell therapy, with the notion that induction of immune responses with CAR $\mathrm{T}$ cells may instigate checkpoint pathways in immunologically cold tumors that would otherwise not respond to ICB. This study gives insights to a mechanism by which CAR T cells and ICB work in synergy to modulate immune landscape of immunologically cold tumors, and our ongoing studies will continue to elucidate the TMEmediated immunosuppression of CAR T cell therapy.

http://dx.doi.org/10.1136/jitc-2021-SITC2021.224 